\title{
POTENCIAIS DE RENDIMENTO DOS ALVOS DA PESCA DE ARRASTO DE TALUDE DO SUDESTE E SUL DO BRASIL ESTIMADOS A PARTIR DE PARÂMETROS DO CICLO DE VIDA
}

\author{
PEREZ, J.A.A. \\ Centro de Ciências Tecnológicas da Terra e do Mar - CTTMar \\ Universidade do Vale do Itajaí - UNIVALI \\ Cx. Postal 360, Itajaí, SC, CEP 88302-202, Brasil. \\ E-mail: angel.perez@univali.br
}

\begin{abstract}
Perez, J.A.A. 2006. Potential yield of trawl fishing targets in southeastern and southern Brazil slope waters as estimated from life-history parameters. Braz. J. Aquat. Sci. Technol. 10(2):1-11. ISSN 1808-7035. Trawl fishing in slope waters of southern and southeastern sectors of Brazilian coast has been sustained by important concentrations of the gulf hake (Urophycis mystacea), argentine hake (Merluccius hubbsi), silver John dory (Zenopsis conchifera), monkfish (Lophius gastrophysus) and, seasonally, the argentine squid (Illex argentinus). Potential yields were estimated for the former four species using growth, natural mortality and selectivity parameters available in the literature or calculated from empirical relationships and length frequency distributions of the trawl catches. These potentials oscillated between 8 and $10 \%$ of the total virginal biomass $\left(B_{o}\right)$ and the total virginal exploitable biomass $\left(E_{x} B_{o}\right)$ for the gulf hake and the argentine hake. In relation to the siver John dory estimated maximum catches corresponded to $12.5 \%$ of $E x B_{o}$, and $9.5 \%$ of $B_{o}$, whereas in the monkfish these catches were limited to $6 \%$ of both $E x B_{o}$, and $B_{o}$. Because the available biomass estimated for these stocks in the slope waters of southeastern and southern Brazil cannot be considered as virginal, at least for the gulf hake and the argentine hake, the estimated potential yields may be generally regarded as conservative reference points. They suggest that precautionary annual catches of the main targets combined (gulf hake, the argentine hake and the silver John dory) should be under $10 \%$ of their total biomass available. Additionally, regarding the monkfish, an important component of the trawl bycatch and the main target of a gill net fishery in the same area, the TAC (Total Allowable Catch) adopted for management purposes is well bellow (2,5\%) the maximum levels estimated by life-history parameters.
\end{abstract}

Keywords: deep-water trawl, Urophycis mystacea, Merluccius hubbsi, Zenopsis conchifera, Lophius gastrophysus

\section{INTRODUÇÃO}

A pesca de arrasto no talude das regiões Sudeste e Sul do Brasil iniciou seu desenvolvimento ao final da década de 1990, como uma das estratégias apresentadas pela numerosa frota de arrasteiros no sentido de compensar as perdas geradas pelo esgotamento dos recursos demersais de plataforma (Perez et al., 2001). A partir da expansão das áreas de pesca para a borda da plataforma esses arrasteiros intensificaram, inicialmente, a captura de recursos valiosos como o lagostim (Metanephrops rubellus), o camarão-cristalino (Plesionika longirostris), o linguado-areia (Paralicthys isósceles, P. triocelata), o congrio-rosa (Genypterus brasiliensis), o peixe-sapo (Lophius gastrophysus) e as arraias emplastro (Atlantorraja spp.) (Perez \& Pezzuto, 1998; Kotas, 1998; Perez et al., 2001; Tomás \& Cordeiro, 2003; Perez et al., 2003a). Em uma segunda etapa, parte dessa frota ocupou os fundos arrastáveis do talude, atividade que foi compartilhada por arrasteiros estrangeiros arrendados para a pesca exploratória so- bre áreas profundas (Perez et al., 2001; Perez et al., 2002a; Perez et al., 2003b). Entre 2001 e 2003, essa pescaria revelou-se estruturada em três estratos batimétricos distintos, cada um definido por alvos específicos e ocupado diferencialmente pelas cerca de 270 embarcações engajadas nessa atividade (Perez \& Pezzuto, 2006).

O estrato "borda de plataforma", entre 100 e 250 $\mathrm{m}$, caracterizou-se por uma pescaria multiespecífica fundamentalmente sustentada por recursos de elevado valor comercial já mencionados acima. Nas áreas mais profundas do "talude inferior", entre 500 e 1000 m de profundidade, a pesca de arrasto foi direcionada principalmente ao recurso camarão-carabinero (Aristeomorpha edwardsiana) (Pezzuto et al., 2006), enquanto que, no estrato batimétrico intermediário ou "talude superior", entre 250 e $500 \mathrm{~m}$ de profundidade, a abrótea-de-profundidade (Urophycis mystacea) e a merluza (Merluccius hubbsi) constituíram os principais alvos de arrasteiros duplos e simples (Perez \& Pezzuto, 2006). Nessa última área também se destacaram o peixe-sapo, o galo- 
de-profundidade (Zenopsis conchifera) e o calamar-argentino (Illex argentinus), sendo que o primeiro foi alvo de uma pescaria dirigida com redes de emalhe (Perez et al., 2002a; Perez \& Wahrlich, 2005), o segundo foi apenas valorizado pelas embarcações arrendadas (Perez et al., 2003) e o último mostrou-se um alvo altamente sazonal, sofrendo um elevado direcionamento nos meses de inverno no sul do Brasil (Perez \& Pezzuto, 2006).

Esforços para o ordenamento da pesca de arrasto de talude, conduzidos desde 2003 em nível do Comitê Consultivo Permanente de Gestão dos Recursos Demersais de Profundidade (CPG/ Demersais) da Secretaria Especial de Aqüicultura e Pesca (SEAP/PR), têm enfocado as três áreas de pesca como unidades de gestão independentes. Dentre esses esforços temse tentado dimensionar uma frota de arrasteiros para a pesca de "talude superior" tomando-se como referência os níveis de abundância e os potenciais de produção da abrótea-de-profundidade, da merluza, galo-deprofundidade e calamar-argentino, além do peixe-sapo que, apesar de ser alvo de pesca direcionada com redes de emalhe de fundo (PEREZ et al., 2002a), é considerado um elemento importante da fauna acompanhante da pesca de arrasto de talude (Perez \& Pezzuto, 2006). Em uma primeira abordagem foi proposta a utilização de uma taxa de remoção de $20 \%$ da biomassa conjunta das três primeiras espécies acima, seguindo os níveis já praticados pela frota entre 2001 e 2003 (Anon, 2005). Embora emergencial, essa taxa de remoção claramente careceu de marcos biológicos referenciais e, portanto, uma revisão da mesma, a partir da análise aprofundada dos potenciais de produção sustentável, tornou-se imprescindível como subsídio para o estabelecimento de Pontos Biológicos de Referência, PBR's (Caddy \& Mahon, 1996), úteis para o ordenamento dessa pescaria.

Potenciais de produção sustentável são comumente estimados a partir da modelagem de séries históricas de captura e esforço. Pescarias em desenvolvimento recente não dispõem dessas séries e, portanto, métodos alternativos devem ser considerados (Garcia et al., 1989). Dentre esses métodos, são particularmente úteis aqueles que relacionam os níveis de produção dos estoques com parâmetros do ciclo de vida, facilmente estimados e freqüentemente disponíveis mesmo para pescarias recentes (Beddington \& Kirkwood, 2005). O presente trabalho apresenta uma revisão desses métodos e explora sua aplicação nos estoques-alvo da pesca de arrasto de talude do Sudeste e Sul do Brasil como subsídio para o estabelecimento de pontos biológicos de referência para o ordenamento dessa pescaria.

\section{MATERIAL E MÉTODOS}

A estimativa de rendimentos potenciais a partir de parâmetros do ciclo de vida

A noção intuitiva de que populações de organismos de vida longa e crescimento lento apresentam um potencial de rendimento sustentável inferior a de populações de organismos de vida curta e rápido crescimento foi pela primeira vez expressa através da equação de Gulland (1971)

$$
R M S=0,5 M B_{0}
$$

onde são combinadas (a) uma consideração teórica de que o rendimento máximo sustentável (RMS) é obtido quando a população atinge a metade da biomassa virginal $\left(B_{o}\right)$, segundo modelo logístico de dinâmica de biomassa e (b) uma consideração observacional que indica que para vários estoques o RMS tende a ser obtido quando da taxa instantânea de mortalidade por pesca $(F)$ é aproximadamente igual à mortalidade natural $(M)$. Desta forma, peixes de crescimento rápido e vida curta, que normalmente apresentam elevadas taxas de mortalidade natural, são aqueles a suportarem maiores rendimentos sustentáveis e vice-versa.

Embora avaliações de estoques tenham, em geral, demonstrado que o RMS deva resultar de uma população com biomassa maior do que $0,5 B_{o}$, análises utilizando parâmetros do ciclo de vida dos estoques indicaram que o rendimento potencial é de fato proporcional à M. Kirkwood et al. (1994), utilizando um modelo populacional estruturado por classes de idade com denso dependência inserida numa relação estoque recrutamento, demonstraram que esta proporcionalidade se estabelece considerando o rendimento como proporção da biomassa virginal e tende a se sustentar tanto em cenários de recrutamento constante como naqueles onde o recrutamento varia como uma função do tamanho do estoque. No primeiro caso partiram da expressão de Rendimento por Recruta definida por Beverton \& Holt (1957):

$$
\begin{aligned}
& Y=F R W_{\infty}\left(1-l_{C}\right)^{\frac{M}{K}}\left(\frac{1}{F+M}-\frac{3\left(1-l_{C}\right)}{F+M+K}+\frac{3\left(1-l_{C}\right)^{2}}{F+M+2 K}-\frac{\left(1-l_{C}\right)^{3}}{F+M+3 K}\right) \\
& E x B_{O}=R W_{\infty}\left(1-l_{C}\right)^{\frac{M}{K}}\left(\frac{1}{M}-\frac{3\left(1-l_{C}\right)}{M+K}+\frac{3\left(1-l_{C}\right)^{2}}{M+2 K}-\frac{\left(1-l_{C}\right)^{3}}{M+3 K}\right)
\end{aligned}
$$


Onde:

$Y$, é o rendimento,

$F$, é a taxa instantânea de mortalidade por pesca,

$R$ é o número de recrutas,

$W_{\infty}$, é o peso assintótico obtido através da equação de crescimento de von Bertalanffy,

$K$ é o parâmetro de curvatura da equação de crescimento de von Bertalanffy,

$I_{c}$ é o comprimento de primeira captura, expresso como uma proporção do parâmetro correspondente ao comprimento assintótico da equação de crescimento de von Bertalanffy

$E x B_{o}$, é a porção explotável da biomassa virginal, ou seja de indivíduos maiores que $I_{c}$.

A razão $Y / E x B_{o}$ produz uma equação de grande complexidade cuja constante de proporcionalidade com $M$ é uma função dos parâmetros $F, M, K$ l $l_{c}$ :

$$
\frac{Y}{E x B_{O}}=M f\left(\frac{F}{M}, \frac{M}{K}, l_{c}\right)
$$

Quando a razão $M / K$ e $I_{c}$ são mantidos fixos no modelo e a função é maximizada em termos de $F$, obtêm-se $R M S$ como proporção de $B_{o}$ sendo então que:

$$
\frac{R M S}{E x B_{O}} \propto M
$$

Um conjunto de possíveis constantes de proporcionalidade foi calculado por Kirkwood et al. (1994) para valores de $M / K$ entre 0,5 e 4,0, segundo estimativas listadas por Pauly (1980), e $I_{c}$ entre 0,2 e 0,6 (Tabela 1). O mesmo conjunto foi igualmente calculado usando a biomassa total $B_{\text {. }}$.

Beddington \& Kirkwood (2005) buscaram uma simplificação das relações acima ressaltando que em particular Mé pouco conhecida, sobretudo em regiões cujas demandas por ordenamento pesqueiro são freqüentemente limitadas pela falta de dados contínuos sobre pescarias e estoques. Nesse sentido utilizaram relações empíricas entre $M, K, t_{m}$ (idade de primeira maturação) e $I_{m}$ (comprimento de primeira maturação) que foram inicialmente conhecidas como os "invariantes de Beverton e Holt" onde:

$$
\begin{aligned}
M t_{m} & =C_{1} \\
\frac{M}{K} & =C_{2}
\end{aligned}
$$

$$
\frac{l_{m}}{l_{\infty}}=C_{3}
$$

e $C_{1}, C_{2}$ e $C_{3}$ são constantes (Beverton \& Holt, 1959). Essas constantes foram inicialmente obtidas empiricamente através da comparação desses parâmetros entre diversos estoques conhecidos. Jensen (1996), entretanto, demonstrou que essas constantes de proporcionalidade não eram apenas obtidas empiricamente, mas podiam ser igualmente obtidas considerando teorias ecológicas que explicam a evolução dos padrões dos ciclos de vida, particularmente as relações compensatórias entre sobrevivência e fecundidade. Essa análise permitiu o cálculo de $C_{1}, C_{2}$ e $C_{3}$ como 1,65, 1,5 e 0,67 respectivamente. Beddington \& Kirkwood (2005) utilizaram essas constantes para simplificar as relações definidas por Kirkwood et al. (1994) de forma a permitir estimativas de rendimentos potenciais através de $\mathrm{Ke} l_{c}$. Em um cenário de recrutamento constante e considerando $\mathrm{M} / \mathrm{K}=1,5$ :

$$
\frac{R M S}{E x B_{O}}=a\left(l_{c}\right) K
$$

onde a constante de proporcionalidade entre $R M S / E x B_{o}$ e $K$ pode ser aproximada pela relação:

$$
\frac{R M S}{E x B_{O}}=0,2 K\left(1-\ln \left(0,67-l_{c}\right)\right)
$$

Nas pescarias onde $I_{c}<I_{m}$ existe também uma relação de proporcionalidade entre $K$ e $F_{\text {max }}$, taxa instantânea de mortalidade por pesca que produz o RMS:

$$
F_{\max }=a\left(l_{c}\right) K
$$

onde $a\left(l_{c}\right)$ pode ser aproximada pela relação:

$$
F_{\max }=\frac{0,6 k}{0,67-l_{c}}
$$

Os autores ressaltam, no entanto, que como o pressuposto de recrutamento constante implica em extrema denso-dependência, $F_{\text {max }}$, estimado a partir dessa relação, quase sempre é maior do que o valor real. Assim, se o valor atual de Fultrapassa $F_{\text {max }}$ é provável que o estoque esteja sobrexplotado.

As relações (4), (10) e (12) acima foram aplicadas aos estoques de merluza, abrótea-de-profundida- 
Tabela 1 - Constantes de proporcionalidade da relação entre $R M S / E x B_{o}, R M S / B_{o}$ e $M$ quando o recrutamento é constante para diferentes valores de $I_{c}$ e $M / K$. Reproduzido de Kirkwood et al. (1994).

\begin{tabular}{lccccc}
\hline \hline$E x B_{0}$ & \multicolumn{5}{c}{$\mathrm{M} / \mathrm{K}$} \\
\cline { 2 - 5 }$I_{c}$ & 0,5 & 1,0 & 2,0 & 3,0 & 4,0 \\
\hline 0,2 & 0,30 & 0,25 & 0,22 & 0,22 & 0,23 \\
0,3 & 0,32 & 0,28 & 0,26 & 0,28 & 0,30 \\
0,4 & 0,35 & 0,32 & 0,33 & 0,36 & 0,42 \\
0,5 & 0,40 & 0,37 & 0,41 & 0,48 & 0,55 \\
0,6 & 0,45 & 0,44 & 0,52 & 0,61 & 0,68 \\
& & & & & \\
$B_{0}$ & & & $M / K$ & & \\
$I_{c}$ & 0,5 & 1,0 & 2,0 & 3,0 & 4,0 \\
\hline 0,2 & 0,30 & 0,25 & 0,22 & 0,21 & 0,22 \\
0,3 & 0,32 & 0,27 & 0,25 & 0,26 & 0,27 \\
0,4 & 0,35 & 0,31 & 0,30 & 0,30 & 0,29 \\
0,5 & 0,39 & 0,35 & 0,33 & 0,31 & 0,28 \\
0,6 & 0,43 & 0,39 & 0,35 & 0,28 & 0,19 \\
\hline \hline
\end{tabular}

de e galo-de-profundidade explotados no talude do Sudeste e Sul do Brasil pela frota de arrasteiros com vistas às estimativas referenciais de $R M S / E x B_{o}, R M S / B_{0}$ e $F_{\text {max }}$. Por simplicidade, todas as análises estiveram vinculadas ao pressuposto de recrutamento constante.

Parâmetros do ciclo de vida dos alvos da pesca de arrasto de talude

A tabela 2 apresenta o conjunto de parâmetros do ciclo de vida levantados na literatura. Sempre que disponíveis, foram utilizados valores de $K e L_{\infty}$ para ambos os sexos juntos. No caso do peixe-sapo optou-se pela utilização dos parâmetros estimados para as fêmeas. Esta opção foi justificada pela diferença marcante encontrada entre os sexos e pela maior abundância das fêmeas nas capturas comerciais. Os mesmos parâmetros foram utilizados para a estimativa da idade dos indivíduos mais velhos presentes nos estoques $\left(T_{\text {max }}\right)$.

O comprimento de primeira captura $\left(I_{c}\right)$ foi definido como aquele que apresenta $50 \%$ de probabilidade de ficar ou não retido na rede de arrasto, segundo um modelo logístico de seletividade (Sparre \& Venema, 1998). Estimativas de $I_{c}$ para os estoques de merluza, abrótea-de-profundidade e galo-de-profundidade foram obtidas através de distribuições de comprimento das espécies constriuídas por observadores a bordo dos arrasteiros estrangeiros arrendados entre 2001 e 2003 e, no caso da abrótea-de-profundidade, das capturas do N/Pq Soloncy Moura (CEPSUL/ IBAMA) durante viagens de pesca científica do calamar-argentino no sul do Brasil entre 2004 e 2005. Para cada espécie estimou-se o número de indivíduos capturados a partir de uma classe de comprimento mínimo (L') acima do qual se assumiu que não haveria possibilidade de escape pela malha do saco da rede de arrasto. Essa estimativa se fez a partir (a) do cálculo da declividade obtida através do ajuste de um modelo exponencial ou potencial ao número de indivíduos capturados acima de L'e (b) da projeção dessa declividade para as classes de comprimento abaixo de L', permitindo a estimativa do número de peixes (captura esperada, $C E_{\text {) }}$ ) da população para essas classes (Figura 1). A proporção de indivíduos retidos em cada classe de comprimento $\left(P_{\mid}\right)$foi calculada através da equação:

$$
P_{l}=\frac{C_{l} / C E_{l}}{\operatorname{Máx}\left[C_{l} / C E_{l}\right]}
$$


Tabela 2 - Parâmetros do ciclo de vida relevantes para a estimativas de potenciais de produção sustentável dos recursos demersais alvos da pesca de arrasto do talude $(250-500 \mathrm{~m})$ no Sudeste e Sul do Brasil.

\begin{tabular}{lllllll}
\hline \hline \multirow{2}{*}{ Merluccius hubbsi } & \multicolumn{1}{c}{$K$} & $L_{\infty}$ & $T_{\max }$ & $L_{m}$ & $I_{c}$ \\
\hline & Machos & $0,350^{1}$ & $475,0^{1}$ & & & \\
& Fêmeas & $0,260^{1}$ & $657,0^{1}$ & & $356,8^{1}$ & \\
& Ambos & $0,230^{1}$ & $697,0^{1}$ & 12 & & 214,1 \\
& & & & & & \\
\hline Urophycis mystacea & Machos & $0,198^{2}$ & $507,3^{2}$ & & & \\
& Fêmeas & $0,154^{2}$ & $698,9^{2}$ & & $439,0^{2}$ & \\
& Ambos & $0,108^{2}$ & $794,6^{2}$ & 14 & & 302,2 \\
& & & & & & \\
\hline Zenopsis conchifera & Machos & & & & & \\
& Fêmeas & & & & & \\
& Ambos & $0,142^{3}$ & $628,7^{3}$ & 15 & $290,5^{5}$ & 344,1 \\
& & & & & & \\
\hline Lophius gastrophysus & Machos & $0,299^{4}$ & $551,4^{4}$ & & $416,0^{4}$ & \\
& Fêmeas & $0,125^{4}$ & $953,6^{4}$ & & $517,0^{4}$ & \\
& Ambos & - & - & 18 & & 270,0 \\
\hline \hline
\end{tabular}

1 Vaz-dos-Santos \& Wongtchowski, 2005; ${ }^{2}$ Haimovici et al., 2005; ${ }^{3}$ Duarte-Pereira, et al., 2005; ${ }^{4}$ Lopes, $2005 ;{ }^{5}$ AbreuSilva et al., 2005.

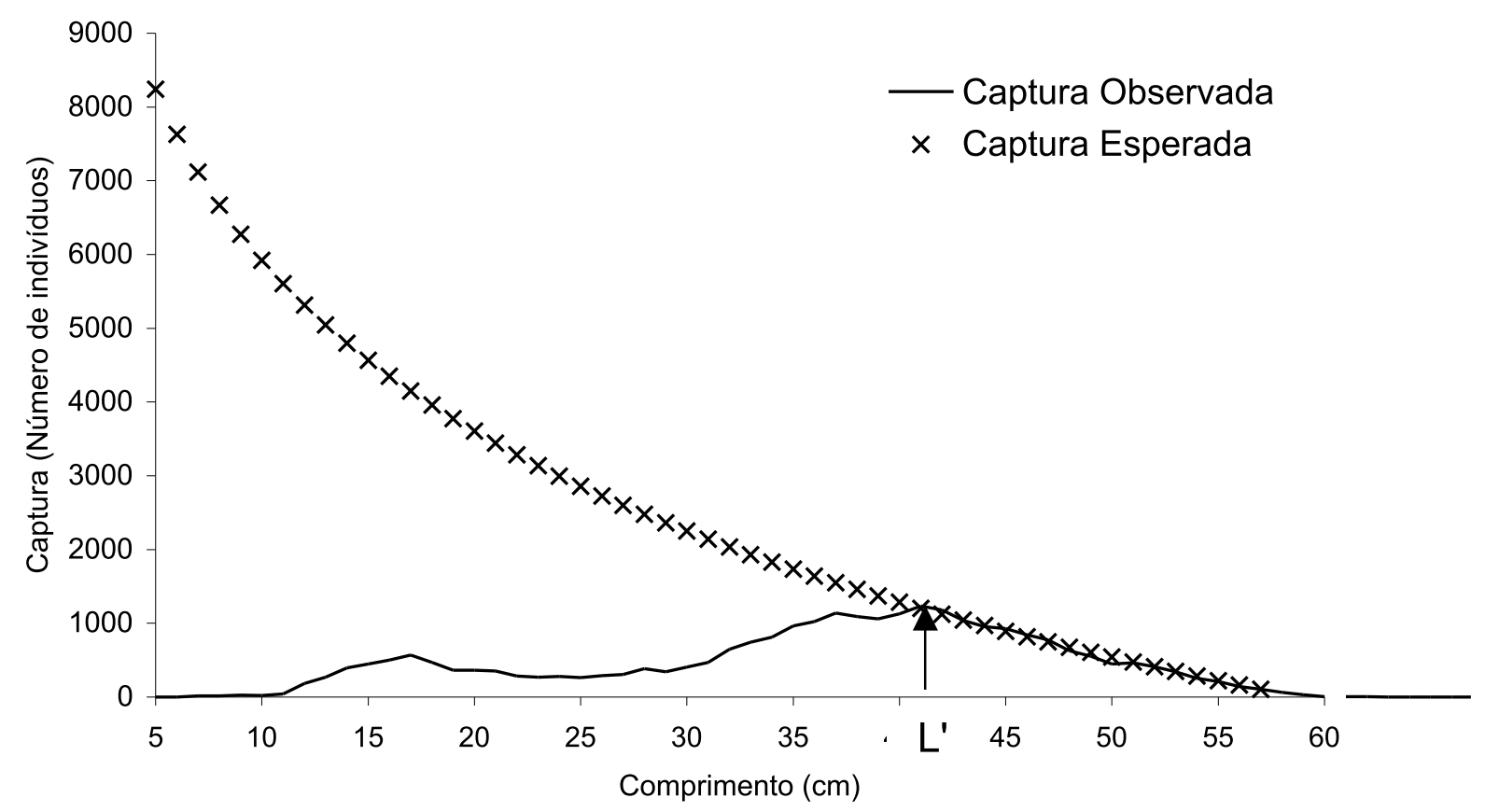

Figura 1 - Exemplo de reconstituição da composição de comprimentos da população a partir da composição de capturas de uma rede de arrasto (Sparre \& Venema, 1998). L', comprimento mínimo de total seletividade pela malha do saco da rede de arrasto. 
onde $C$, é a captura observada para a classe de comprimento i. Os modelos logísticos:

$$
\begin{gathered}
P_{l}=\frac{1}{\left(1+e^{p 1-p 2 \cdot l}\right)} \\
P_{l}=\frac{1}{\left(1+e^{p 1-p 2 \cdot l}+e^{p 3-p 4 \cdot l}+e^{p 1+p 3-(p 2+p 4) l}\right)}
\end{gathered}
$$

foram tentativamente ajustados às proporções $P_{\text {, obti- }}$ das pelo procedimento acima através da estimativa dos parâmetros $p 1, p 2, p 3$ e $p 4$ por interação até a minimização da soma dos quadrados dos resíduos (SSQ). O modelo escolhido foi aquele que resultou no menor SSQ e $I_{c}$ foi calculado pela relação:

$$
l_{c}=\frac{p 1}{p 2}
$$

Os $I_{c}$ estimados (Tabela 2, Figura 2) foram expressos como proporção do $L_{\infty}$ (Tabela 3 ).

As estimativas da taxa instantânea de mortalidade natural $M$ foram obtidas através das relações empíricas de Hoenig (1983) que utilizam a longevidade, representada por $T_{\max }$ (Tabela 2) para predizer $M$. No caso da merluza e do peixe-sapo foram incluídas alguns valores de $M$ encontrados na literatura (Tabela 3 ).

\section{RESULTADOSE DISCUSSÃO}

$R M S / E x B_{o}$, e $R M S / B_{o}$ dos estoques merluza, abrótea, galo-de-profundidade e peixe-sapo foram calculados considerando-se as constantes de proporcionalidade entre essas variáveis e $M$ (Tabela 1) e os valores de Mestimados para essas espécies (Tabela 3). Nas Figuras 3 a 6 apresentam-se possíveis valores de $R M S / E x B_{o}$, e $R M S / B_{o}$ obtidos a partir da fixação de $K$ e da variação de $M$ e $I_{c}$. Observa-se que para conjunto abrótea-de-profundidade (Figura 4), galode-profundidade (Figura 5) e peixe-sapo (Figura 6) o potencial de explotação sustentável deve se situar entre 5 e $18 \%$ da biomassa virginal explotável e entre 4 e $10 \%$ da biomassa virginal. A merluza diferencia-se dessas espécies apresentando potenciais relativamente mais elevados, possivelmente situados entre 10 e $30 \%$ da biomassa virginal explotável e entre 9 e 18\% da biomassa virginal (Figura 3).

Quando valores $M, K$ e $I_{c}$ estimados para o Sudeste e Sul do Brasil (Tabela 1) são aplicados utilizando-se as mesmas constantes de proporcionalidade acima (Tabela 3) observa-se que os potenciais de explotação sustentável estão restritos aos limites infe- riores apresentados nas figuras 3 a 6 , oscilando entre 8 e $10 \%$, tanto para $E x B_{o}$, quanto para $B_{o}$ no caso da merluza e da abrótea-de-profundidade, atingindo 12,5\% de $E x B_{o}$, e 9,5\% de $B_{o}$ no caso do galo-de-profundidade e em torno de $6 \%$ para $E x B_{o}$, e $B_{o}$ no caso do peixesapo.

O galo-de-profundidade apresenta-se com potencial de explotação ligeiramente mais elevado basicamente em função de ter sua captura aparentemente concentrada na parcela de indivíduos relativamente maiores. Já o peixe-sapo é o que apresenta a menor mortalidade natural de todos os quatro recursos, sendo que, se considerarmos os níveis ainda menores uti-
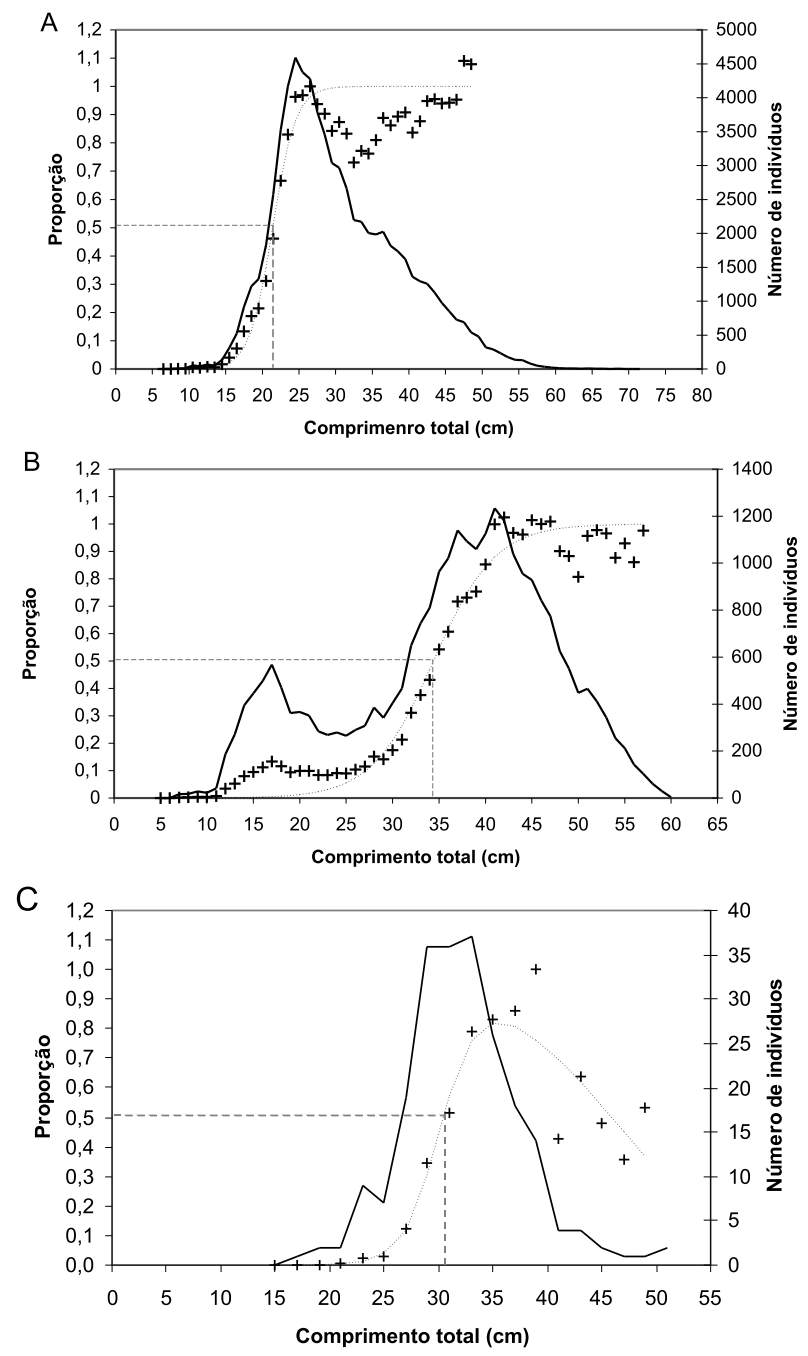

Figura 2 - Análise para determinação do comprimento de primeira captura $I_{c}$ pela rede de arrasto dos recursos merluza Merluccius hubbsi $(\mathrm{A})$, galo-de-profundidade Zenopsis conchifera $(\mathrm{B})$ e abrótea-de-profundidade Urophycis mystacea (C). Linha contínua, composição observada de comprimentos da captura; cruzes, proporções de indivíduos retidos em cada classe de comprimento $P_{l}$; linha pontilhada, modelo logístico ajustado $P_{l}$ A posição de $I_{c}$ está indicada pela $P_{I}=0,5$. 
lizados por Perez et al. (2005), obtém-se estimativas em torno de $4 \%$ tanto para $E x B_{0}$, quanto para $B_{0}$

Paralelamente à abordagem acima, $E x B_{o}$ foi calculado utilizando a relação simplificada (10) (Tabela 3) onde se observam discrepâncias em relação aos recursos abrótea-de-profundidade, galo-de-profundidade e peixe-sapo. Nesses casos, os valores estimados de M/K aproximam-se de 2 afastando-se da "invariante" 1,5 assumido por Beddington \& Kirkwood (2005). Além disso, nas espécies de crescimento mais lento, a abrótea-de-profundidade e o peixe-sapo, as estimativas do rendimento potencial se reduzem para 4,8 e 4,9\% de $E x B_{0}$, respectivamente.

Os níveis máximos de mortalidade por pesca $F_{\max }$ que permitiriam a obtenção do $R M S$ também puderam ser estimados a partir da relação simplificada (12), com a restrição de que $I_{c}<I_{m}$. Como o galo-de-profundidade não atendeu essa restrição (Tabela 2), a estimativa de $F_{\max }$ não foi possível nessa espécie. Vaz-dos-Santos \& Rossi-Wongtschowski (2005) estimaram, para a merluza, uma taxa instantânea de mortalidade total $Z$ de 0,95 ano-1 para ambos os sexos entre 1996 e 2002. Considerando-se $M=0,37 \mathrm{ano}^{-1}$, o valor correspondente de $F=0,58$ ano $^{-1}$ encontra-se claramente acima de $F_{\max }$ apontando para um cenário de sobrepesca desse estoque (Tabela 3). Da mesma forma, Haimovici et al.
(2005) estimaram os valores $F$ para machos e fêmeas da abrótea-de-profundidade do estoque Sul em 0,50 e 0,40 ano $^{-1}$, respectivamente, e para machos e fêmeas do estoque Sudeste em 0,32 e 0,39 ano- $^{-1}$, respectivamente. Em todos os casos, estes valores ultrapassam as estimativas de $F_{\max }$ obtidas a partir dos parâmetros do ciclo de vida (Tabela 3). Finalmente, Perez et al. (2005) analisaram valores de $F$ de peixe-sapo por classe de comprimento demonstrando que, acima de $I_{c}$, a pesca em 2001 praticou valores entre 0,1 e 0,2 ano $^{-1}$, ou seja próximos de $F_{\text {max }}$, apenas elevando-se em comprimentos maiores que $60 \mathrm{~cm}$. A julgar pela queda na abundância do estoque após esse ano é possível que $F_{\max }=0,19$ possa de fato ser uma sobre-estimação.

Os métodos aplicados nesta análise às espécies-alvo da pesca de arrasto de talude, permitem a obtenção de estimativas do rendimento e mortalidade por pesca potenciais dessas espécies a partir de parâmetros básicos do ciclo de vida já disponíveis. Ressalta-se, no entanto, que o uso das relações empíricas simplificadas propostas por Beddington \& Kirkwood (2005) requer maior precaução uma vez que existem indícios de sobre-estimação dos pontos referências para algumas espécies como o $F_{\text {max }}$ por exemplo. De fato, Walters \& Martel (2002) ressaltam que o valor de $\mathrm{F}$ não deveria ultrapassar $0,5 \mathrm{M}-0,6 \mathrm{M}$, o que,

Tabela 3 - Estimativa dos potenciais de produção sustentável da merluza (Merluccius hubbsi), abrótea-de-profundidade (Urophycis mystacea), galo-de-profundidade (Zenopsis conchifera) e peixe-sapo (Lophius gastrophysus) a partir de parâmetros do ciclo de vida no Sudeste e Sul do Brasil.

\begin{tabular}{|c|c|c|c|c|c|c|c|c|c|}
\hline \multirow[b]{2}{*}{ Espécie } & \multirow[b]{2}{*}{$M$} & \multirow[b]{2}{*}{$\mathrm{M} / \mathrm{K}$} & \multirow[b]{2}{*}{$I_{c}$} & \multicolumn{4}{|c|}{ Desconsiderando invariantes } & \multicolumn{2}{|c|}{$\begin{array}{c}\text { Considerando } \\
\text { invariantes }\end{array}$} \\
\hline & & & & $\begin{array}{c}\text { Constante } \\
\text { ExB。 }\end{array}$ & $\begin{array}{c}\text { Constante } \\
\mathrm{B}_{\mathrm{o}}\end{array}$ & $\begin{array}{l}\text { RMS/ } \\
\text { ExB }_{0}\end{array}$ & $\begin{array}{c}\mathrm{RMS} / \\
\mathrm{B}_{0}\end{array}$ & $\mathrm{RMS} / \mathrm{B}_{\mathrm{o}}$ & $F_{\max }$ \\
\hline \multirow{2}{*}{$\begin{array}{c}\text { Merluccius } \\
\text { hubbsi }\end{array}$} & 0,37 & 1,61 & 0,31 & 0,272 & 0,262 & 0,101 & 0,097 & 0,093 & 0,383 \\
\hline & $0,30^{1}$ & 1,30 & & 0,266 & 0,256 & 0,080 & 0,077 & & \\
\hline $\begin{array}{l}\text { Urophycis } \\
\text { mystacea }\end{array}$ & 0,30 & 1,9 & 0,38 & 0,316 & 0,290 & 0,095 & 0,087 & 0,048 & 0,223 \\
\hline $\begin{array}{l}\text { Zenopsis } \\
\text { conchifera }\end{array}$ & 0,30 & 2,1 & 0,55 & 0,417 & 0,317 & 0,125 & 0,095 & 0,089 & - \\
\hline \multirow[t]{2}{*}{$\begin{array}{c}\text { Lophius } \\
\text { gastrophysus }\end{array}$} & 0,25 & 2,0 & $0,28^{2}$ & 0,252 & 0,244 & 0,063 & 0,061 & 0,049 & 0,192 \\
\hline & $0,15^{3}$ & 1,2 & & 0,264 & 0,254 & 0,040 & 0,038 & & \\
\hline
\end{tabular}

${ }^{1}$ Cordo et al., 2004; ${ }^{3}$ Schwingel \& Andrade, 2002; ${ }^{3}$ Perez et al., 2005 

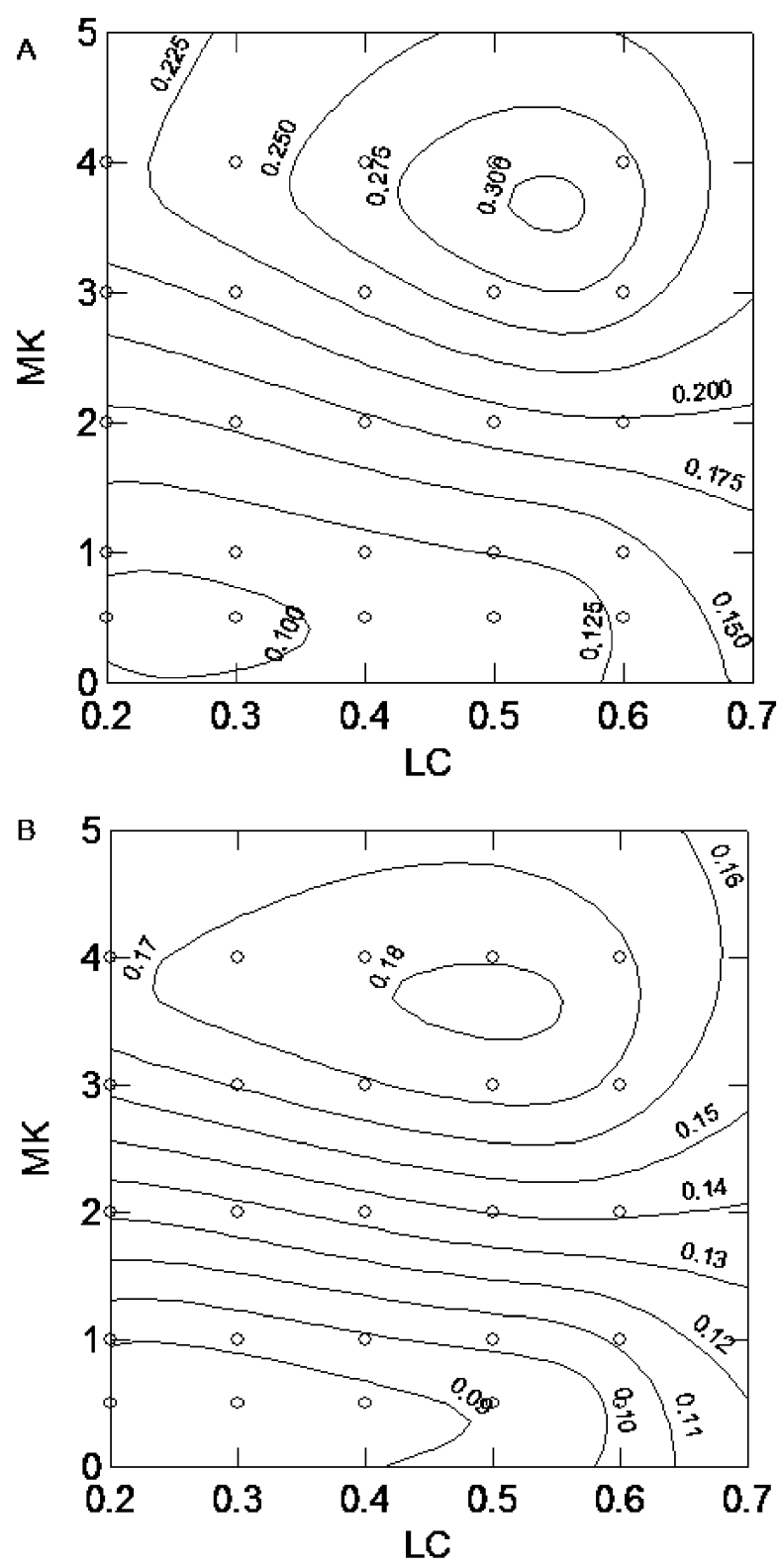

Figura 3 - Estimativas de Rendimento Máximo Sustentável (RMS) como proporção da (A) Biomassa Virginal Explotável $\left(E B_{0}\right)$ e $(B)$ Biomassa Virginal $\left(\mathrm{B}_{\mathrm{o}}\right.$ ) para a merluza (Merluccius hubbsi) a partir das relações de proporcionalidade com a taxa instantânea de mortalidade natural $\mathrm{M}$. Os valores foram calculados para um valor fixo de $\mathrm{K}=0,230$.

considerando as estimativas $F_{\text {max }}$ neste estudo, parece ser o caso da merluza, abrótea-de-profundidade e o peixe-sapo. A mesma tendência de sobre-estimação não parece ter sido evidenciado nos resultados obtidos com o procedimento proposto por Kirkwood et al. (1997) o que sugere uma ferramenta útil para a revisão crítica das taxas de remoção propostas em 2005 (Anon, 2005) e para o dimensionamento da frota atuante nessa área do talude brasileiro.
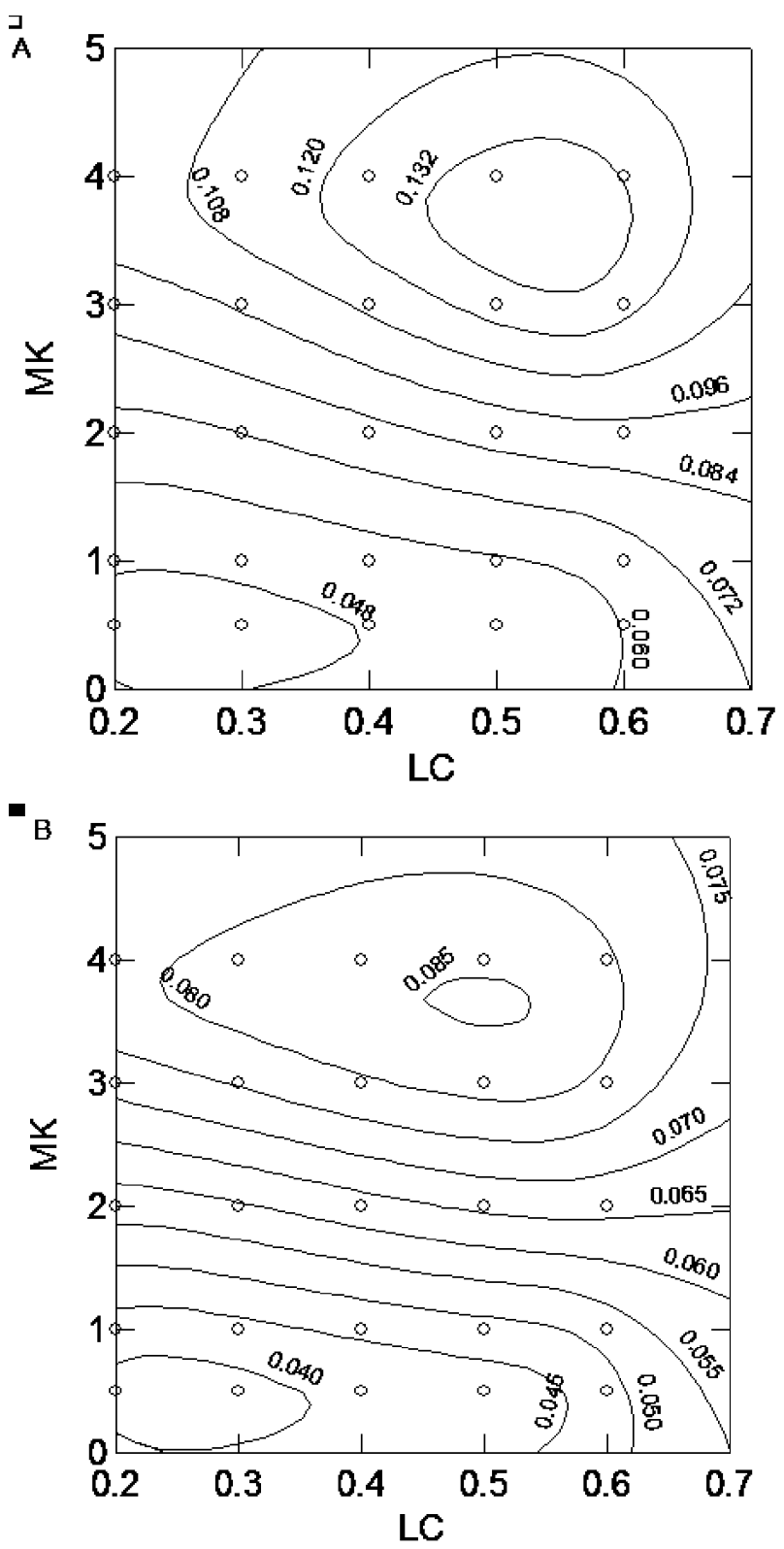

Figura 4 - Estimativas de Rendimento Máximo Sustentável (RMS) como proporção da (A) Biomassa Virginal Explotável $\left(E B_{0}\right)$ e (B) Biomassa Virginal $\left(\mathrm{B}_{\mathrm{o}}\right.$ ) para a abrótea-de-profundidade (Urophycis mystacea) a partir das relações de proporcionalidade com a taxa instantânea de mortalidade natural M. Os valores foram calculados para um valor fixo de $\mathrm{K}=0,108$.

Parece evidente que para as três espécies-alvo (merluza, abrótea-de-profundidade e galo-de-profundidade), as taxas de remoção não deveriam ultrapassar em torno de $10 \%$ da biomassa virginal. No entanto, devese considerar que as estimativas de biomassa disponíveis já não representem a biomassa virginal, visto que, em particular a abrótea-de-profundidade e a merluza, sofreram exploração comercial anterior ao período estudado. Ainda assim essas taxas poderiam ser consi- 

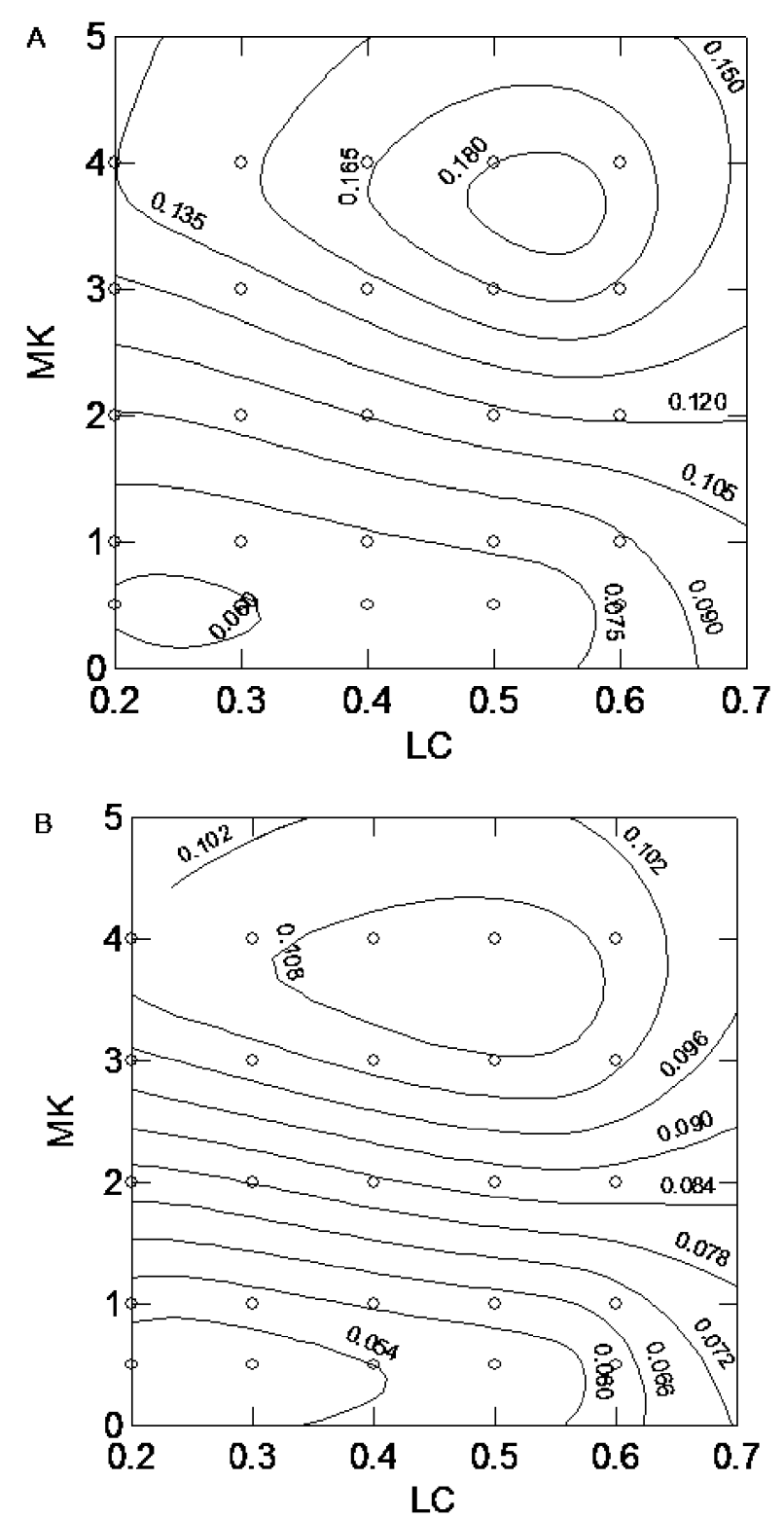

Figura 5 - Estimativas de Rendimento Máximo Sustentável (RMS) como proporção da (A) Biomassa Virginal Explotável (ExB $)$ e (B) Biomassa Virginal $\left(\mathrm{B}_{0}\right)$ para o galo-de-profundidade (Zenopsis conchifera) a partir das relações de proporcionalidade com a taxa instantânea de mortalidade natural M. Os valores foram calculados para um valor fixo de $\mathrm{K}=0,108$.

deradas referências conservativas (pois 10\% da biomassa atual representam uma captura menor que $10 \%$ da biomassa virginal) podendo balizar o dimensionamento da captura e esforço nessas áreas.

No que se refere ao peixe-sapo, a espécie é alvo da pescaria específica com o emprego de redes de emalhe de fundo e a taxa máxima de explotação estimada no presente estudo poderia auxiliar os níveis referenciais já utilizados (Perez et al., 2002b). Segun-
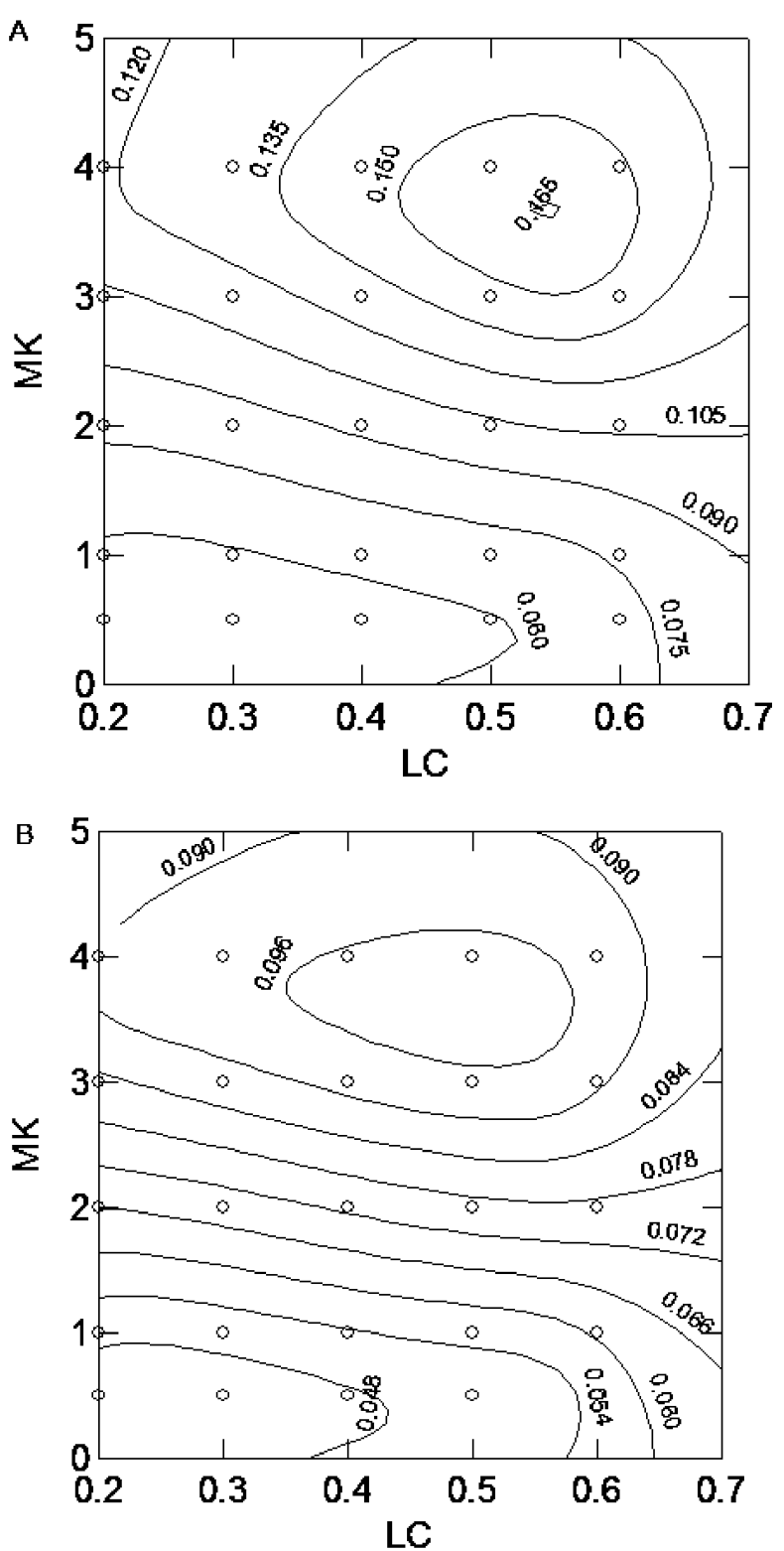

Figura 6 - Estimativas de Rendimento Máximo Sustentável (RMS) como proporção da (A) Biomassa Virginal Explotável (ExBo $)$ e (B) Biomassa Virginal $\left(\mathrm{B}_{0}\right)$ para a peixe-sapo (Lophius gastrophysus) a partir das relações de proporcionalidade com a taxa instantânea de mortalidade natural $\mathrm{M}$. Os valores foram calculados para um valor fixo de $K=0,125$.

do esses níveis, a taxa de remoção exercida pela cota máxima de captura $(\mathrm{RMS}=2.500 \mathrm{t})$, proposta por Perez et al. (2005), estaria em torno de $4 \%$ da biomassa virginal, ou seja, compatível com os limites previstos nesta análise. Em 2002, o SCC CPG/ Demersais (Anon, 2002) reduziu essa cota para $1.500 \mathrm{t}$ em função de evidentes decréscimos populacionais nas áreas de pesca, o que levou a uma taxa de remoção de $2,5 \%$ da biomassa virginal. Essa cota não tem sido cumprida (Anon, 2006) 
e, portanto, pode-se prever que as capturas encontramse acima dos níveis de sustentabilidade. A mesma conclusão pode ser extraída das estimativas de $F_{\text {max }}$ em relação aos estoques de merluza e abrótea-de-profundidade.

\section{AGRADECIMENTOS}

O presente estudo faz parte das atividades previstas no Convênio "Programa de Apoio Técnico e Científico ao Desenvolvimento da Pesca no Sudeste e Sul do Brasil" celebrado entre a Universidade do Vale do Itajaí e a Secretaria Especial de Aqüicultura e Pesca da Presidência da República (SEAP/ PR/64/2005). Os resultados aqui apresentados foram avaliados na 4ㄹ $\mathrm{Reu-}$ nião Ordinária do Subcomitê Científico do Comitê Consultivo Permanente de Gestão dos Recursos Demersais de Profundidade (CPG/Demersais), realizada em Itajaí, de 3 a 5 de maio de 2006, e serviram de subsídios para as recomendações técnicas de ordenamento dessas pescarias. O autor é grato a todos os membros do SCC/ CPG pelos comentários e críticas assim como de Humber Agrelli de Andrade pela ajuda na aplicação dos modelos matemáticos.

\section{REFERÊNCIAS}

Abreu-Silva, J.L.; Duarte-Pereira, M. \& Andrade, H.A. 2005. Maturação e estrutura de comprimento do peixe-galo-de-profundidade Zenopsis conchifera (Lowe, 1852) capturado no talude continental do sul e sudeste do Brasil. II Congresso Brasileiro de Oceanografia-CBO'2005, 09-12 de outubro de 2005, Vitória, ES. Livro de resumos.

Anon, 2002. Relatório da $2^{\mathrm{a}}$ Reunião Ordinária. Comitê Consultivo Permanente de Gestão dos Recursos Demersais de Profundidade. Subcomitê Científico. Ministério da Agricultura, Pecuária e Abastecimento (MAPA). Itajaí, SC, 11 - 12 de dezembro de 2002. 34p.

Anon, 2005. Relatório da $3^{a}$ Reunião Ordinária. Comitê Consultivo Permanente de Gestão dos Recursos Demersais de Profundidade. Subcomitê Científico. Secretaria Especial de Aqüicultura e Pesca da Presidência da República (SEAP/PR). Itajaí, SC, 14 16 de março de 2005. 34p.

Anon, 2006. Relatório da $4^{\mathrm{a}}$ Reunião Ordinária. Comitê Consultivo Permanente de Gestão dos Recursos Demersais de Profundidade. Subcomitê Científico. Secretaria Especial de Aqüicultura e Pesca da Presidência da República (SEAP/PR). Itajaí, SC, 3 - 5 de maio de 2006. 56p.
Beddington, J.R. \& Kirkwood, G.P. 2005. The estimation of potential yield and stock status using life-history parameters. Phil. Trans. R. Soc. B. 360: 163-170.

Beverton, R.J.H. \& Holt, S.J. 1957. On the dynamics of exploited fish populations. MAFF Fish. Invest. Lond. Ser. 2. 19: 1-533.

Beverton, R.J.H. \& Holt, S.J. 1959. A review of the life spans and mortality rates of fish in nature and the relationship to growth and other physiological characteristics. Ciba Found. Colloq. Age. 5:142 177.

Caddy, J.F.\& Mahon, R. 1996. Puntos de referencia para la ordenación pesquera. FAO Documento Técnico de Pesca, 347.

Cordo, H.D.; Renzi, M.\& Hirusta, G. 2004. Evaluación del estado del efectivo norte de $41^{\circ} \mathrm{S}$ de la merluza (Merluccius hubbsi) y estimación de la captura biológicamente aceptable correspondiente al año 2004. Informe Técnico Interno INIDEP. No. 33, 28p.

Duarte-Pereira, M.; Abreu-Silva, J.L.; Ebert, L.A \& Andrade, H.A. 2005. Idade e crescimento do peixe galo-de-profundidade, Zenopsis conchifera (Lowe, 1852) capturado na plataforma e talude continental do sul e sudeste do Brasil: dados preliminares. II Congresso Brasileiro de Oceanografia-CBO'2005, $09-12$ de outubro de 2005, Vitória, ES. Livro de resumos

Garcia, S.; Sparre, P. \& Csirke, J. 1989. Estimating surplus production and Maximum Sustainable Yield from biomass data when catch and effort series are not available. Fish. Res. 8: 13-23.

Gulland, J.A. 1971. The fish resources of the ocean. Fishing News (Books) Limited Publishers, West Byfleet, Surrey.

Haimovici, M.; Ávila-da-Silva, A. O. \& Fischer, L.G. 2005. Urophycis mystacea (Ribeiro, 1903). Relatório apresentado na $3^{\mathrm{a}}$ Reunião do Sub Comitê Científico do Comitê Consultivo Permanente de Gestão de Recursos Demersais de Profundidade (CPG/ Demersais) - SEAP/PR. DOC 18 SCC CPG 03 2005, 8p.

Hoenig, J.M., 1983. Empirical use of longevity data to estimate mortality rates. Fish. Bull. 82:898-903.

Jensen, A.L. 1996. Beverton and Holt life history invariants result from optimal trade-off of reproduction and survival. Can. J. Fish. Aquat. Sci. 53: 829 822.

Kirkwood, G.P.; Beddington, J.R. \& Rossouw, J.R. 1994. Harvesting species of different lifespans. In: Largescale ecology and conservation biology (ed. P.J. Edwards, R. May \& N.R. Webb) pp. 199-227. Oxford: Blackwell Scientific.

Kotas, J.E. 1998. Fauna acompanhante nas pescarias de camarão em Santa Catarina. Coleção Meio 
Ambiente. Série Estudos Pesca 24. MMA, IBAMA, $75 \mathrm{p}$.

Lopes, F.R.A. 2005. Reprodução, idade e crescimento e do peixe-sapo Lophius gastrophysus (Ribeiro, 1915) na Região Sudeste e Sul do Brasil. Dissertação de Mestrado. Programa de Pós-graduação em Ciência e Tecnologia Ambiental, Universidade do Vale do Itajaí - UNIVALI, 68p.

Pauly, D. 1980. On the interrelationships between natural mortality, growth parameters, and mean environmental temperature in 175 fish stocks. J. Cons. CIEM. 39(2): 175-192.

Perez, J.A.A. \& Pezzuto, P.R. 1998. Valuable shellfish species in the by-catch of shrimp fishery in southern Brazil: spatial and temporal patterns. J. Shell. Res., 17(1):303-309.

Perez, J.A.A. \& Pezzuto, P.R. 2006. A pesca de arrasto de talude do Sudeste e Sul do Brasil: Tendências da frota nacional entre $2001-2003$. Bol. Inst. Pesca, São Paulo, 32(2): 127-150.

Perez, J.A.A.; Pezzuto, P.R. \& Andrade, H.A 2005. Biomass assessment of the monkfish Lophius gastrophysus stock exploited by a new deep-water fishery in southern Brazil. Fish. Res. 72: 149-162.

Perez, J.A.A. \& Wahrlich, R. 2005. A bycatch assessment of the gillnet monkfish Lophius gastrophysus fishery off southern Brazil. Fish. Res. 72: 81-95.

Perez, J.A.A.; Pezzuto, P.R.; Rodríguez, L.F.; Valentini, H. \& Vooren, C.M. 2001. Relatório da reunião técnica de ordenamento da pesca de arrasto nas regiões Sudeste e Sul do Brasil. In: Pezzuto, P.R.; Perez, J.A.A.; Rodríguez, L.F. \& Valentíni, H. (eds.) Reuniões de ordenamento da pesca demersal nas regiões Sudeste e Sul Notas Téc. FACIMAR. 5: 134.

Perez, J.A.A.; Wahrlich, R.; Pezzuto, P. R. \& Lopes, F. R. A. 2002a. Estrutura e dinâmica da pescaria do peixe-sapo Lophius gastrophysus no Sudeste e Sul do Brasil. Boletim do Instituto de Pesca, São Paulo, 28(2):204-231.

Perez, J.A.A.; Pezzuto, P. R.; Andrade, H.A.; Schwingel, P.R.; Rodrigues-Ribeiro, M. \& Wahrlich, R. 2002b. O ordenamento de uma nova pescaria direcionada ao peixe-sapo (Lophius gastrophysus) no Sudeste e Sul do Brasil. Notas Téc. FACIMAR, 6:65-83.
Perez, J.A.A.; Pezzuto, P.R.; Lucato, S.H. \& Vale, W.G. 2003a. Frota de arrasto de Santa Catarina. In: Cergole, M.C. \& Rossi-Wongtchwoski, C.L.D.B. (ed.) Dinâmica das frotas pesqueiras. Análise das principais pescarias comerciais do sudeste-sul do Brasil São Paulo: Evoluir. 117-184 p.

Perez, J.A.A.; Wahrlich, R.; Pezzuto, P.R.; Schwingel, P.R.; Lopes, F.R.A. \& Rodrigues-Ribeiro, M. 2003b. Deep-sea fishery off southern Brazil: recent trends of the Brazilian fishing industry. J. Northwest Atlantic Fish. Sci. 31:1-18.

Perez, J.A.A.; Pezzuto, P.R. \& Andrade, H.A. 2005. Biomass assessment of the monkfish Lophius gastrophysus stock exploited by a new deep-water fishery in southern Brazil. Fish. Res. 72: 149-162.

Pezzuto, P.R.; Perez, J.A.A. \& Wahrlich, R. 2006. Deepsea shrimps (Decapoda: Aristeidae): new targets of the deep-water trawling fishery in Brazil. Brazilian Journal of Oceanography. 54(2-3):123-134.

Schwingel, P.R. \& Andrade, H. A. 2002. Capítulo 3. Aspectos Biológicos e Populacionais. In: Análise da Pescaria do Peixe-Sapo no Sudeste e Sul do Brasil. Convênio UNIVALI - MAPA. Relatório Final (MAPA/ SARC/ DPA/ 03/ 2001), abril, 2002.

Sparre, P. \& Venema, S.C. 1998. Introduction to tropical fish stock assessment. FAO Fisheries Technical Paper. Rome. No. 306/1. Rev. 2: 407p.

Tomas, A.R.G. \& Cordeiro, R.A. 2003. Dinâmica da frota de arrasto de portas do estado do Rio de Janeiro. In: Cergole, M.C. \& Rossi-Wongtschowski, C.L.D.B. Dinâmica das frotas pesqueiras. Análise das principais pescarias comerciais do sudeste-sul do Brasil São Paulo: Evoluir. 15-37 p.

Vaz-dos-Santos, A. \& Rossi-Wongtschowski, C.L.D.B. 2005. Merluccius hubbsi Marini, 1993. In: Análise das Principais Pescarias Comerciais da região Sudeste-Sul do Brasil: dinâmica populacional das espécies em explotação. (eds. M.C. Cergole; A.O. Ávila-da-Silva; C.L.D.B Rossi-Wongtschowski). Série Documentos REVIZEE: Score Sul. pp: 8893.

Walters, C. \& Martell, S.J.D. 2002. Stock assessment needs for sustainable fisheries. Bull. Mar. Sci. 70(2):629-638. 